\title{
Expression and promoter methylation analysis of ATP-binding cassette genes in pancreatic cancer
}

\author{
MINYONG CHEN $^{1 *}$, XIAOFENG XUE $^{1 *}$, FEITONG WANG $^{1}$, YONG AN $^{1}$, DONG TANG $^{1}$, YUE XU $^{1}$, \\ HUI WANG ${ }^{1}$, ZHONGXU YUAN $^{1}$, WENTAO GAO ${ }^{2}$, JISHU WEI $^{2}$, JINGJING ZHANG $^{3}$ and YI MIAO ${ }^{2}$ \\ ${ }^{1}$ Laboratory of General Surgery, and ${ }^{2}$ Department of Surgery, The First Affiliated Hospital \\ of Nanjing Medical University; ${ }^{3}$ Jiangsu Province Academy of Clinical Medicine Institute \\ of Tumor Biology, 300 Guangzhou Road, Nanjing 210029, P.R. China
}

Received July 3, 2011; Accepted August 29, 2011

DOI: $10.3892 /$ or.2011.1475

\begin{abstract}
We investigated the relationship of $A B C B 1 / M D R 1$, $A B C C 1 / M R P 1$ and $A B C G 2 / B C R P$ expression and promoter methylation with pancreatic cancer tumorigenesis and drug resistance. Gemcitabine-resistant pancreatic cancer cells, SW1990/ GZ (33.3-fold increased resistance), were obtained by treating SW1990 cells with gemcitabine. The expression of $A B C B 1 /$ $M D R 1, A B C C 1 / M R P 1$ and $A B C G 2 / B C R P$ was determined by quantitative real-time RT-PCR in the cell lines, 3 normal pancreatic tissues, 15 human pancreatic cancer samples and 15 adjacent tissues. Promoter methylation was determined in cell lines by bisulfite genomic sequencing. ABCB1/MDR 1, ABCC1/ $M R P 1$ and $A B C G 2 / B C R P$ were upregulated in SW1990 and SW1990/GZ compared with normal pancreatic tissue, and expression in SW1990/GZ was significantly higher than in SW1990 cells. $A B C B 1 / M D R 1, A B C C 1 / M R P 1$ and $A B C G 2 /$ $B C R P$ were upregulated in pancreatic cancer tissues, compared to adjacent tissues. The $A B C B 1 / M D R 1, A B C C 1 / M R P 1$ and $A B C G 2 / B C R P$ promoter were hypomethylated in all the cell lines. ABCB1/MDR1, ABCC1/MRP1 and ABCG2/BCRP expression correlated with pancreatic cancer tumorigenesis and drug resistance in a mechanism that is independent of promoter methylation.
\end{abstract}

\section{Introduction}

Pancreatic cancer is among the 10 leading causes of cancerrelated deaths, with a 5 -year survival $<5 \%$ (1). Most patients have advanced stage disease at diagnosis. One fifth of pancre-

Correspondence to: Dr Yi Miao, Department of General Surgery, The First Affiliated Hospital of Nanjing Medical University, 300 Guangzhou Road, Nanjing 210029, P.R. China

E-mail: miaoyi@njmu.edu.cn

*Contributed equally

Key words: ATP-binding cassette genes, ABCB1/MDR1, ABCC1/ MRP1, ABCG2/BCRP, DNA methylation, drug resistance, pancreatic cancer atic cancer patients present in the early stage and are eligible for surgical resection (2), which is a complex operation that is associated with high rates of operative morbidity. Pancreatic cancer also shows resistance to chemotherapy. Gemcitabine, the standard treatment in advanced disease, prolongs survival by 5-6 months. Chemotherapy is largely ineffective for patients with metastases as disease frequently redevelops even after surgery; therefore, there is an urgent need to understand the molecular mechanism of chemoresistance in pancreatic cancer.

One well-known mechanism of chemoresistance is the overexpression of energy-dependent pumps, such as adenosine triphosphate-binding cassette (ABC) transporters, which lead to the extrusion of drugs and their metabolites (3). P-gp, a 170,000-Da phosphoglycoprotein that consists of two ATP binding cassettes and two transmembrane regions, which is associated with chemoresistance in cancers of the gastrointestinal tract, liver and kidneys (4). The breast cancer resistance protein (ABCG2/BCRP) is a 655-amino-acid polypeptide transporter with a wide range of substrates associated with breast cancer resistance (5). Additionally, ABCC1/MRP1 is correlated with differentiation grade and tumor size in primary hepatocellular carcinoma and breast cancer $(6,7)$.

DNA methylation of $\mathrm{CpG}$ dinucleotides is the predominant epigenetic gene expression modification. More than $40 \%$ of protein coding genes have $5^{\prime} \mathrm{CpG}$ rich segments, termed CpG islands (8). The aberrant methylation of gene promoters is frequently observed in human cancers and correlates with carcinogenesis, tumor progression and chemosensitivity $(9,10)$. Methylation of gene promoters is inversely proportional to transcriptional activity (11). Epigenetic silencing is mediated via methyl $\mathrm{CpG}$ binding proteins (MBDs) which act as docking sites for co-repressor proteins such as histone deacetylases, histone methyltransferases (12). MDR1 mRNA transcription is inversely correlated with DNA methylation in various cancers, including prostate, gastric carcinomas $(10,13)$. Human tumors often display altered DNA methylation, including both genome-wide hypomethylation and site-specific hypermethylation (14).

Demethylation of $\mathrm{CpG}$ islands in the $A B C B 1 / M D R l$ promoter region is a mechanism of chemoresistance, which induces the expression of P-gp and the multidrug-resistant (MDR) phenotype (15). The objectives of this study were to investigate the 
expression and $\mathrm{CpG}$ island methylation status of ATP-binding cassette genes in pancreatic cancer, and the possible molecular mechanisms of carcinogenesis and chemosensitivity.

\section{Materials and methods}

Tissue samples and cell lines. Fifteen pairs of samples (15 pancreatic cancer tissues, 15 adjacent tissues $3-5 \mathrm{~cm}$ from the cancer edge) and three normal pancreatic tissues were obtained from patients suffering from pancreatic ductal adenocarcinoma and patients with pancreatic injury (experimental procedures manipulating the human tissues or human-derived cells met the principles of Declaration of Helsinki and were approved by the Ethics Committee of the First Affiliated Hospital of Nanjing Medical University). Each sample was cryopreserved in liquid nitrogen. The human pancreatic cancer cell line SW1990 was obtained from the Shanghai Cell Bank (Shanghai, China). Gemcitabine-resistant pancreatic cancer cells, SW1990/GZ, were obtained by treating the parental cell line SW1990 with increasing doses (maximum dose $200 \mu \mathrm{mol} / \mathrm{l}$ ) of gemcitabine intermittently for 24 weeks to generate stable cultures. All cell lines were cultured in DMEM (Invitrogen, Carlsbad, CA, USA) supplemented with $10 \%$ fetal bovine serum (FBS; Sigma, St. Louis, MO, USA), $2 \mathrm{mM}$ glutamine, $100 \mu \mathrm{g} / \mathrm{ml}$ penicillin, and $100 \mu \mathrm{g} / \mathrm{ml}$ streptomycin at $37^{\circ} \mathrm{C}$ in a humidified chamber supplemented with $5 \% \mathrm{CO}_{2}$.

Quantitative real-time RT-PCR assays for gene expression analysis. Total RNA was isolated using the Qiagen RNeasy Mini Kit (Qiagen, Shanghai, China) according to the manufacturer's protocol and quantified by absorption at $260 \mathrm{~nm} . A B C B 1$, $A B C C 1$ and $A B C G 2$ real-time RT-PCR primers were listed in Table I. Quantitative RT-PCR was performed using an ABI PRISM 7000 Sequence Detection System (Applied Biosystems, Foster City, CA) with SYBR Premix Ex Taq (Takara, Dalian, China). Relative gene expression was normalized to the internal reference gene, glucose-6-phosphate dehydrogenase (GAPDH).

DNA methylation analysis. DNA was isolated using the Omega Tissue DNA kit (Omega, Shanghai, China) and 500 ng was subjected to bisulfite conversion using the EpiTect Bisulfite Kit (Qiagen) according to the manufacturer's instructions and prepared for PCR amplification using the Takara Taq Kit (Takara). Primers for the bisulfite-treated gene promoters (Table II) were designed using Methyl Primer Express software v1.0 (Applied Biosystems). The PCR amplification products were extracted from 2\% agarose gels using the Gel Extraction Kit (Omega), cloned into the pEASY-T1 simple vector (TransGen Biotech, Beijing, China) and transfected into Trans1-T1 phage-resistant chemically competent cells according to the manufacturer's instructions. The bacterial fluid containing pEASY-T1 target DNA was spread on solid LB medium, incubated overnight at $37^{\circ} \mathrm{C}$ and bacterial clones were picked into $1.0 \mathrm{ml}$ liquid LB medium and sent to Invitrogen Corporation Shanghai Representative Office for sequencing.

Statistical analysis. Results were expressed as the mean \pm SEM of at least triplicate measurements. The Student's t-test was used to compare values with SPSS 13.0 software, and $p<0.05$ was considered statistically significant.
Table I. $A B C B 1, A B C C 1$ and $A B C G 2$ real-time RT-PCR primers.

\begin{tabular}{|c|c|c|}
\hline Gene & Primers & $\operatorname{Tm}\left({ }^{\circ} \mathrm{C}\right)$ \\
\hline \multicolumn{3}{|l|}{$\mathrm{ABCB} 1$} \\
\hline Sense & 5'-CGAAGCAGTGGTTCAGGTG-3' & 60.0 \\
\hline Antisense & 5'-TGTGATCCACGGACACTCC-3' & \\
\hline \multicolumn{3}{|l|}{ ABCG2 } \\
\hline Sense & 5'-GAAGAGTGGCTTTCTACCTT-3' & 61.0 \\
\hline Antisense & 5'-GTCCCAGGATGGCGTTGA-3' & \\
\hline \multicolumn{3}{|l|}{$\mathrm{ABCC} 1$} \\
\hline Sense & 5'-CGCCTTCGCTGAGTTCCT-3' & 62.5 \\
\hline Antisense & 5'-TGCGGTGCTGTTGTGGTG-3' & \\
\hline
\end{tabular}

Table II. $A B C B 1, A B C C 1$ and $A B C G 2$ promoter bisulfite genomic sequencing primers.

\begin{tabular}{|c|c|c|}
\hline Gene & Primers & $\begin{array}{ll}\text { Size } & \mathrm{Tm} \\
(\mathrm{bp}) & \left({ }^{\circ} \mathrm{C}\right)\end{array}$ \\
\hline
\end{tabular}

\section{$\mathrm{ABCB} 1$}

Sense 5'-GTAGTGGTATTGGATTATGTTG-3'

Antisense 5'-TTAATACCCCAACTACTCTAAC-3' $260 \quad 60$

$\mathrm{ABCG} 2$

Sense 5'- AATGAGYGTTTGGTGATTTT-3'

Antisense 5'- ATTTCCCCAAATCRAAATTC-3'

$256 \quad 60$

$\mathrm{ABCC} 1$

Sense 5'-GTTTAAGTTTTGGAGGATTTGG-3'

Antisense 5'-CAACRCCRCCTAATTAACCC-3'

$176 \quad 60$

\section{Results}

Morphological analysis and RI (resistance index) of SW1990/ GZ cells. Parental SW1990 cells have a polygonal and uniform morphology (Fig. 1A). During induction of gemcitabine resistance, cells have a flat elongated morphology, with increased foot processes, with vacuoles, membrane rupture and fuzzy boundaries (Fig. 1B). In SW1990/GZ, reduced cell vacuoles and foot processes were observed in tightly packed cells (Fig. 1C). The IC50 for gemcitabine, the value at which growth was inhibited by $50 \%$, was $100 \mu \mathrm{mol} / 1$ in SW1990/GZ cells and $3.0 \mu \mathrm{mol} / 1$ in SW1990 cells. The RI (resistance index) of SW1990/GZ (IC50 of SW1990/GZ divided by the IC50 of SW1990) is 33.3.

ATP-binding cassette gene mRNA expression is increased in SW199/GZ and SW1990 compared with the normal pancreas tissue. Expression of the ATP-binding cassette genes $A B C B 1$, $A B C C 1$ and $A B C G 2$ was significantly increased in SW1990/ GZ and SW1990 cells compared with the normal pancreatic tissues (Fig. 2). Additionally, expression of $A B C B 1, A B C C 1$ and $A B C G 2$ was significantly higher in SW1990/GZ cells than SW1990 cells $(\mathrm{p}<0.05)$. These results indicate a relationship between ATP-binding cassette gene expression and tumorigenesis and drug resistance in pancreatic cancer cells. 


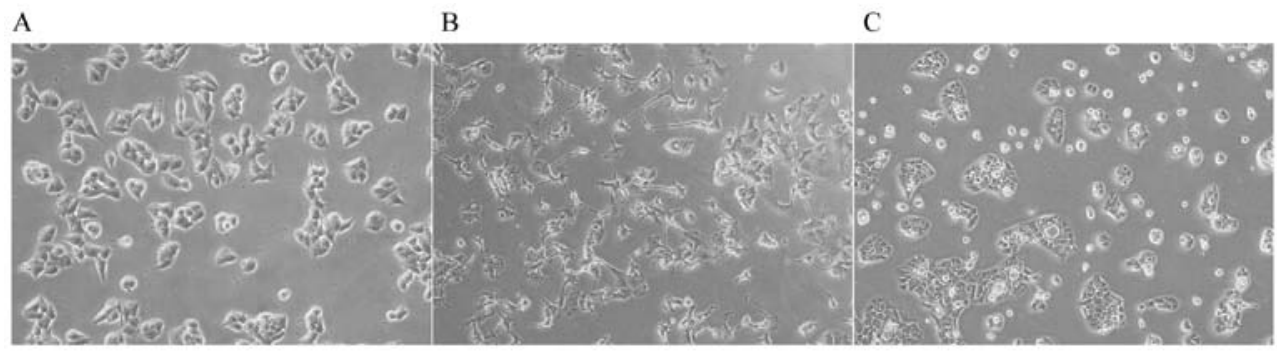

Figure 1. Pancreatic cell line morphology. (A) Parental sw1990 cells; (B) sw1990 cells in the process of gemcitabine induced resistance; (C) sw1990-GZ gemcitabine-resistant cells, $\mathrm{x} 40$.
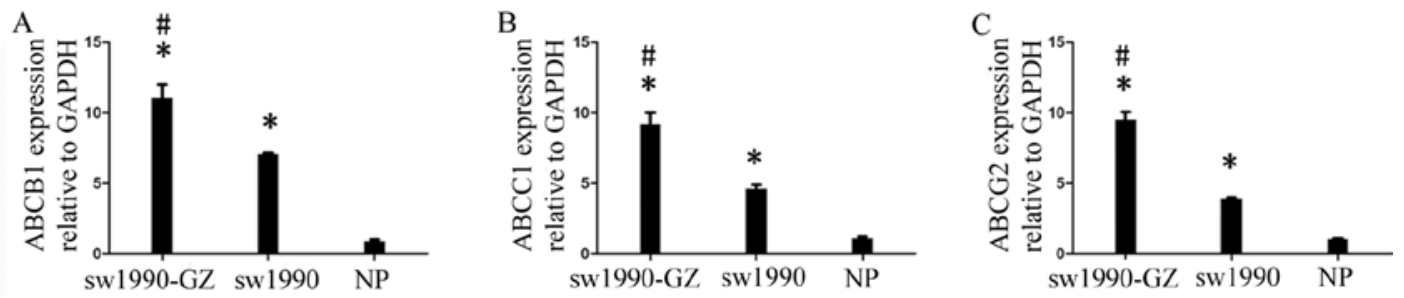

Figure 2. Quantitative real-time PCR for the normal pancreatic tissues (NP), sw1990, and sw1990-GZ gemcitabine-resistant cell lines. (A) ABCB1, (B) ABCC1 and (C) $A B C G 2$ mRNA expressed normalized to GAPDH, ${ }^{*}$ p $<0.05$ vs. NP, ${ }^{*} \mathrm{p}<0.05$ vs. sw1990 cells.

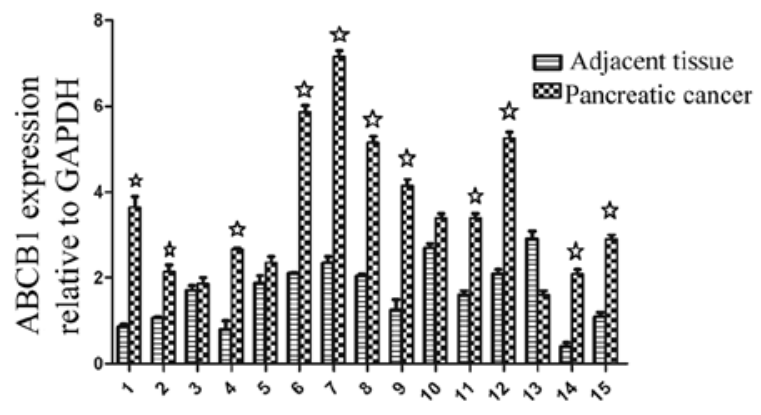

Figure 3. Quantitative real-time PCR of the ATP-binding cassette gene $A B C B 1 /$ $M D R 1$ in pancreatic cancer and matched adjacent tissues for 15 individuals, ${ }^{*} \mathrm{p}<0.05$ vs. adjacent tissue.

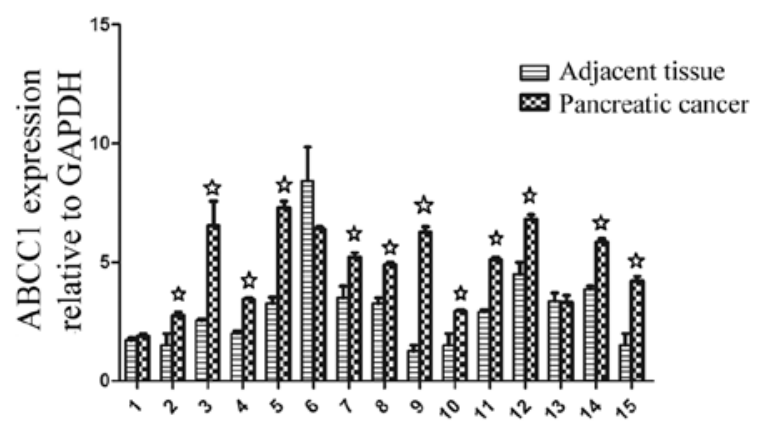

Figure 4. Quantitative real-time PCR of the ATP-binding cassette gene ABCC1/ $M R P 1$ in pancreatic cancer and matched adjacent tissues for 15 individuals, ${ }^{*} \mathrm{p}<0.05$ vs .adjacent tissue.

ATP-binding cassette gene mRNA expression is increased in pancreatic cancer compared to adjacent tissue. Significantly higher $A B C B 1$ mRNA expression was observed in tumors compared to adjacent tissues in $73 \%$ of patients $(11 / 15, \mathrm{p}<0.05$; Fig. 3). Twelve out of $15(80 \%)$ patients had significantly higher

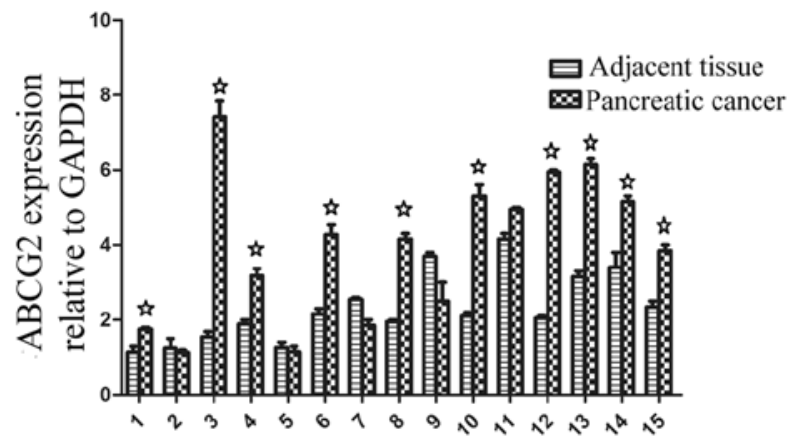

Figure 5. Quantitative real-time PCR of the ATP-binding cassette gene ABCG2/ $B C R P$ in pancreatic cancer and matched adjacent tissues for 15 individuals, ${ }^{*} \mathrm{p}<0.05$ vs. adjacent tissue.

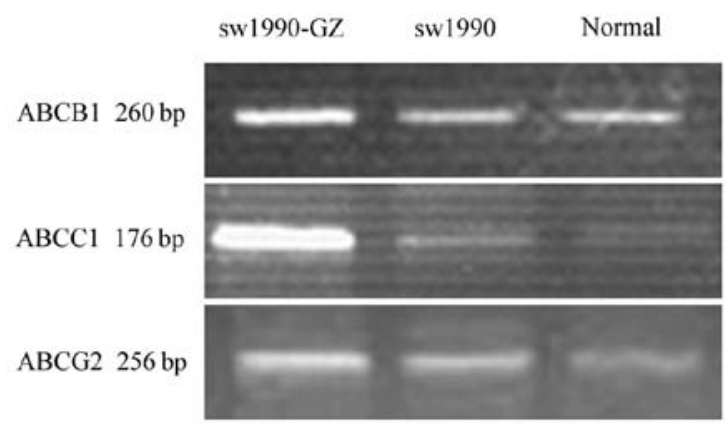

Figure 6. $A B C B 1, A B C C 1$ and $A B C G 2$ promoter bisulfite PCR amplification in sw1990-GZ gemcitabine-resistant, sw1990 and normal pancreatic tissue.

levels of $A B C C 1$ mRNA in tumors than the adjacent tissues (p<0.05; Fig. 4), and $67 \%$ of samples (10/15) showed increased levels of $A B C G 2 \mathrm{mRNA}$ expression in pancreatic cancer tissue samples compared to adjacent tissues ( $p<0.05$, Fig. 5). These 

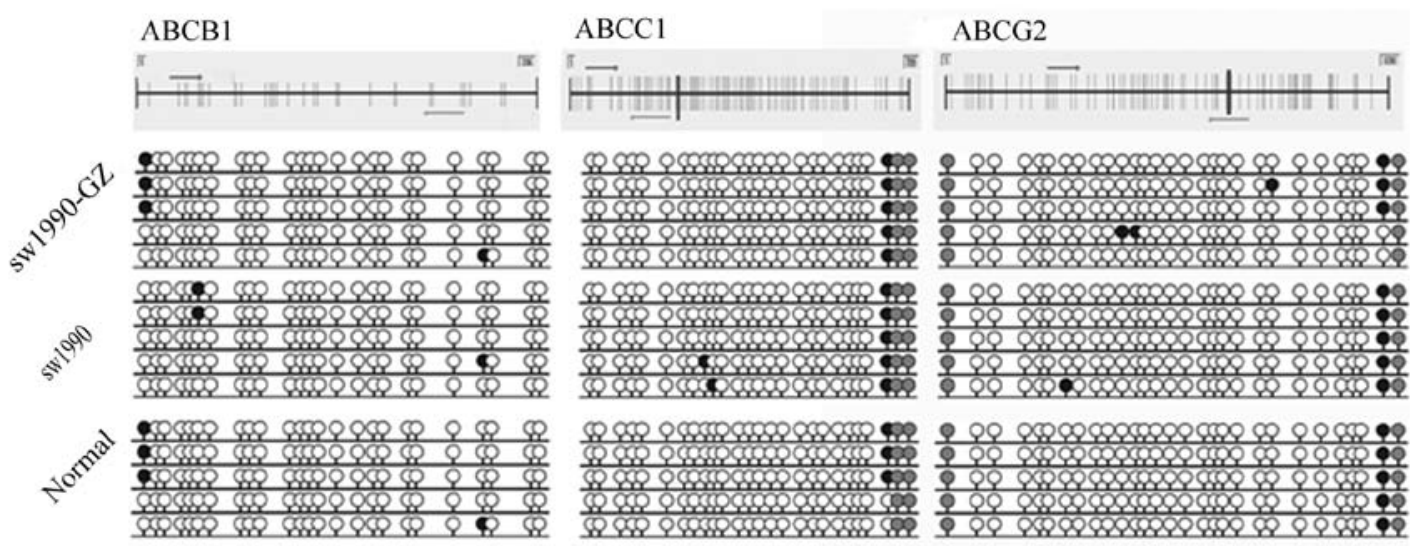

Figure 7. $A B C B 1, A B C C 1$ and $A B C G 2$ promoter methylation status in normal pancreatic tissues, sw1990 and sw1990-GZ gemcitabine-resistant cell lines determined by bisulfite sequencing of five cloned PCR products. Arrows represent promoter bisulfite genomic sequencing primers, open circles indicate unmethylated $\mathrm{CpG}$ sites, filled circles indicate methylated $\mathrm{CpG}$ sites.

results suggest that the expression of ATP-binding cassette genes is related to tumorigenesis in pancreatic cancer.

$A B C B 1, A B C C 1$ and ABCG2 ATP-binding cassette gene promoters are hypomethylated in cell lines. Bisulfite analysis was performed on the $A B C B 1, A B C C 1$ and $A B C G 2$. The bisulfite conversion PCR products (ATP-binding cassette gene promoters) are shown in Fig. 6. The methylation status of the promoters are shown in Fig. 7. The $A B C B 1, A B C C 1$ and $A B C G 2$ promoters were hypomethylated with no significant difference observed between SW1990/GZ, SW1990 and the normal pancreatic tissue. These results indicate that the expression of ATP-binding cassette genes in human pancreatic cancer cells was not correlated with promoter demethylation.

\section{Discussion}

In this study, we compared $\mathrm{ABC}$ transporter gene expression in pancreatic cancer and normal pancreas to determine the mechanisms regulating $\mathrm{ABC}$ transporter expression. Increased expression of $A B C B 1, A B C C 1$ and $A B C G 2$ in pancreatic cancer compared to adjacent tissues, and also in SW1990 cells compared to normal pancreatic tissues, suggested that ATP-binding cassette genes may play an important role in pancreatic cancer oncogenesis. Additionally, the increased expression of ATP-binding cassette genes in gemcitabineresistant cells demonstrated that these genes may contribute to pancreatic cancer drug resistance.

The ATP-binding cassette genes have been shown to be overexpressed in a subpopulation of pancreatic cancer cells with stem cell characteristics, which play an important role in oncogenesis, development and drug resistance (16-18). $A B C B 1$, $A B C C 1$ and $A B C G 2$ expression were altered in a similar manner, which indicated that ATP-binding cassette genes play a joint role in oncogenesis and drug resistance.

No difference in promoter methylation status was observed in SW1990 and SW1990/GZ cells, which indicated that chemotherapy agents do not induce ATP-binding cassette gene methylation in pancreatic cancer. As the methylation status of the three ATP-binding cassette genes was not significantly different in normal pancreatic tissues, SW1990 and SW1990/
GZ cells, promoter methylation does not appear to regulate the expression of ATP-binding cassette genes. Based on the literature and our experimental results, we suggest that alternative mechanisms, such as histone modifications and microRNAs-mediated regulation of gene expression, may regulate ATP-binding cassette genes expression in pancreatic cancer tumorigenesis and drug resistance.

The major epigenetic mechanisms include DNA methylation, histone modifications and RNA-mediated gene silencing. Histone acetylation is important for the maintenance of unmethylated promoter regions and to protect against DNA methylation (19). Promoter hypomethylation is associated with histone acetylation and demethylation (20). Furthermore, histone modifications are a prerequisite for DNA methylation (21). Treatment with depsipeptide or trichostatin A, which are specific histone deacetylase inhibitors, alters DNA methylation status (20). We suggest that histone acetylation of ATP-binding cassette gene promoters may play a more important role in their activation of gene expression in pancreatic cancer than promoter methylation.

The other possible mechanism is regulation of gene expression by microRNAs, small 19-25 nucleotide single-stranded RNAs which regulate gene expression and play an important role in many cellular processes, including development, differentiation, cell proliferation and apoptosis (22) and also drug resistance (23). MiR-21 and miR-27a are overexpressed in pancreatic cancer (24). MiR-21 upregulates MDRl/ABCB1 leading to chemoresistance in breast cancer (25), and anti-miR-21 enhances pancreatic cancer cell chemosensitivity $(26,27)$. MiR-27a is overexpressed in MDR cancer cells, and the inhibition of miR-27a reduces $P$ - $g p$ and MDRI mRNA expression in ovarian cancer and esophageal carcinoma $(28,29)$. Three microRNAs (hsa-miR-328, 519c and 520h) target $A B C G 2$, and their expression negatively correlates with BRCP/ABCG2 expression in both breast cancer and glioblastoma (30,31). Hsa-miR-520h downregulates $A B C G 2$ in pancreatic cancer cells, inhibiting migration, invasion and side population development (32). MiR-326 and miR-134 modulate chemoresistance via $M R P 1 / A B C C 1$ expression $(33,34)$. On the basis of this evidence, we hypothesize that the altered expression of miR-21/miR-27a, hsa-miR-328/-519c/-520h and miR-326/miR-134, which regulate the expression of $A B C B 1 /$ 
$M D R 1, B R C P / A B C G 2, M R P / A B C C 1$, respectively, may lead to pancreatic cancer tumorigenesis and drug resistance.

\section{References}

1. Jemal A, Siegel R, Ward E, et al: Cancer statistics, 2009. CA Cancer J Clin 59: 225-249, 2009.

2. Yeo TP, Hruban RH, Leach SD, et al: Pancreatic cancer. Curr Probl Cancer 26: 176-275, 2002.

3. Gottesman MM, Fojo T, Bates SE, et al: Multidrug resistance in cancer: role of ATP-dependent transporters. Nat Rev Cancer 2: 48-58, 2002

4. Ambudkar SV,Kimchi-Sarfaty C,Sauna ZE, et al: P-glycoprotein: from genomics to mechanism. Oncogene 22: 7468-7485, 2003.

5. Fletcher JI, Haber M, Henderson MJ, et al: ABC transporters in cancer: more than just drug efflux pumps. Nat Rev Cancer 10 $147-156,2010$.

6. Vander Borght S, Komuta M, Libbrecht L, et al: Expression of multidrug resistance-associated protein 1 in hepatocellular carcinoma is associated with a more aggressive tumour phenotype and may reflect a progenitor cell origin. Liver Int 28: 1370-1380, 2008.

7. Filipits M, Suchomel RW, Dekan G, et al: MRP and MDR1 gene expression in primary breast carcinomas. Clin Cancer Res 2 : 1231-1237, 1996.

8. Egger G, Liang G, Aparicio A, et al: Epigenetics in human disease and prospects for epigenetics therapy. Nature 429: 457-463, 2004

9. To KK, Zhan Z, Bates SE, et al: Aberrant promoter methylation of the ABCG2 gene in renal carcinoma. Mol Cell Biol 26: 8572-8585, 2006.

10. Enokida H, Shiina H, Igawa M, et al: $\mathrm{CpG}$ hypermethylation of MDR1 gene contributes to the pathogenesis and progression of human prostate cancer. Cancer Res 64: 5956-5962, 2004.

11. Jones PA and Baylin SB: The fundamental role of epigenetic events in cancer. Nat Rev Genet 3: 415-428, 2002.

12. Fuks F, Hurd PJ, Wolf D, et al: The methyl-CpG-binding protein MeCP2 links DNA methylation to histone methylation. Biol Chem 278: 4035-4040, 2003.

13. Tahara $\mathrm{T}$, Arisawa $\mathrm{T}$, Shibata $\mathrm{T}$, et al: Effect of promoter methylation of multidrug resistance 1 (MDR1) gene in gastric carcinogenesis. Anticancer Res 29: 337-341, 2009.

14. Eden A, Gaudet F, Waghmare A, et al: Chromosomal instability and tumors promoted by DNA hypomethylation. Science 300 $455,2003$.

15. Baker EK, Johnstone RW, Zalcberg JR, et al: Epigenetic changes to the MDR1 locus in response to chemotherapeutic drugs Oncogene 24: 8061-8075, 2005.

16. Du Z, Qin R, Wei C, et al: Pancreatic cancer cells resistant to chemoradiotherapy rich in 'stem-cell-like' tumor cells. Dig Dis Sci 56: 741-750, 2011

17. Wang YH, Li F, Luo B, et al: A side population of cells from a human pancreatic carcinoma cell line harbors cancer stem cell characteristics. Neoplasma 56: 371-378, 2009.

18. Zhou J, Wang CY, Liu T, et al: Persistence of side population cells with high drug efflux capacity in pancreatic cancer. World J Gastroenterol 14: 925-930, 2008.
19. Mutskov VJ, Farrell CM, Wade PA, et al: The barrier function of an insulator couples high histone acetylation levels with specific protection of promoter DNA from methylation. Genes Dev 16: 1540-1554, 2006

20. Vaissière T, Sawan $C$ and Herceg Z: Epigenetic interplay between histone modifications and DNA methylation in gene silencing. Mutat Res 659: 40-48, 2008.

21. Tamaru H and Selker EU: A histone H3 methyltransferase controls DNA methylation in Neurospora crassa. Nature 414: 277-283, 2001.

22. Bartel DP: MicroRNAs: genomics, biogenesis, mechanism and function. Cell 116: 281-297, 2004.

23. Zheng T, Wang J, Chen X, et al: Role of microRNA in anticancer drug resistance. Int J Cancer 126: 2-10, 2010.

24. Bloomston M, Frankel WL, Petrocca F, et al: MicroRNA expression patterns to differentiate pancreatic adenocarcinoma from normal pancreas and chronic pancreatitis. JAMA 297: 1901-1908, 2007.

25. Bourguignon LY, Spevak CC, Wong G, et al: Hyaluronan-CD44 interaction with protein kinase $\mathrm{C}$ (epsilon) promotes oncogenic signaling by the stem cell marker Nanog and the production of microRNA-21, leading to down-regulation of the tumor suppressor protein PDCD4, anti-apoptosis, and chemotherapy resistance in breast tumor cells. J Biol Chem 284: 26533-26546, 2009.

26. Moriyama T, Ohuchida K, Mizumoto K, et al: MicroRNA-21 modulates biological functions of pancreatic cancer cells including their proliferation, invasion, and chemoresistance. $\mathrm{Mol}$ Cancer Ther 8: 1067-1074, 2009.

27. Hwang JH, Voortman J, Giovannetti E, et al: Identification of microRNA-21 as a biomarker for chemoresistance and clinical outcome following adjuvant therapy in resectable pancreatic cancer. PLoS One 5: 10630-10642, 2010.

28. Zhua $\mathrm{H}$, Wu H, Liu X, et al: Role of MicroRNA miR-27a and miR-451 in the regulation of MDR1/P-glycoprotein expression in human cancer cells. Biochem Pharmacol 76: 582-588, 2008.

29. Zhang H, Li M, Han Y, et al: Down-regulation of miR-27a might reverse multidrug resistance of esophageal squamous cell carcinoma. Dig Dis Sci 55: 2545-2551, 2010.

30. Li X, Pan YZ, Seigel GM, et al: Breast cancer resistance protein BCRP/ABCG2 regulatory microRNAs (hsa-miR-328, $-519 \mathrm{c}$ and $-520 \mathrm{~h})$ and their differential expression in stem-like ABCG2+ cancer cells. Biochem Pharmacol 81: 783-92, 2011.

31. Li WQ, Li YM, Tao BB, et al: Downregulation of ABCG2 expression in glioblastoma cancer stem cells with miRNA-328 may decrease their chemoresistance. Med Sci Monit 16: 27-30, 2010.

32. Wang F, Xue X, Wei J, et al: hsa-miR-520h downregulates ABCG2 in pancreatic cancer cells to inhibit migration, invasion, and side populations. Br J Cancer 103: 567-574, 2010.

33. Liang Z, Wua H, Xia J, et al: Involvement of miR-326 in chemotherapy resistance of breast cancer through modulating expression of multidrug resistance-associated protein 1. Biochem Pharmacol 79: 817-824, 2010.

34. Guo L, Liu Y, Bai Y, et al: Gene expression profiling of drugresistant small cell lung cancer cells by combining microRNA and cDNA expression analysis. Eur J Cancer 6: 1692-1702, 2010. 